\title{
Utilidad de la ecotomografía Doppler en el diagnóstico de la arteritis de células gigantes
}

\author{
Joaquín Hevia $\mathbf{M}^{1,2,3 *}$, Jorge González $\mathbf{M}^{4}$.
}

1. Médico radiólogo, Servicio de Radiología, Hospital San Juan de Dios de La Serena. La Serena, Chile.

2. Servicio de Radiología, Clínica Red Salud Elqui. La Serena, Chile.

3. Profesor adjunto, Programa de especialidad en Radiología. Oficina de Postgrado. Facultad de Medicina, Universidad Católica del Norte. Coquimbo, Chile.

4. Residente de radiología, Universidad Católica del Norte. Coquimbo, Chile.

\section{Utility of the Doppler Ultrasound in the Diagnostic Approach of Giant Cell Arteritis}

Resumen: La arteritis de células gigantes es una vasculitis sistémica frecuente, especialmente en mayores de 50 años. Ante la sospecha clínica el diagnóstico debe ser confirmado con histología o estudios de imágenes. La biopsia de arteria temporal se considera el gold standard, sin embargo, tiene sus limitaciones, una de ellas es la alta tasa de falsos negativos. El estudio por ecotomografía Doppler permite la visualización de las arterias temporales y en manos experimentadas puede constituir un procedimiento diagnóstico alternativo a la biopsia en el estudio inicial de esta entidad. Esta técnica es de fácil acceso, bajo costo, sin riesgos y no expone al paciente a radiación ionizante. En este trabajo revisamos la utilidad clínica de la ecotomografía Doppler en el estudio de la arteria temporal y sus hallazgos imagenológicos en la aproximación diagnóstica a la arteritis de células gigantes, siendo las características de compromiso el engrosamiento parietal arterial hipoecogénico, habitualmente concéntrico, no compresible.

Palabras claves: Arteritis de células gigantes, Ecotomografía, Polimialgia reumática, Vasculitis de grandes vasos.

\begin{abstract}
Giant cell arteritis is a frequent systemic vasculitis, especially in patients older than 50 years old. When clinically suspected, the diagnosis should be made on the basis of histology or imaging methods. Temporal artery biopsy is considered the gold standard for the diagnosis but it has some caveats, especially the existence of false negatives. Doppler ultrasound study is a low cost, accessible tool that allows visualization of temporal arteries and, in experienced hands, can replace the biopsy in the initial evaluation of the disease, without the risks of ionizing radiation. In this article we review the clinical utility of the temporal artery Doppler ultrasound and its findings in the diagnostic approach of giant cell arteritis, being characteristic a non-compressible, hypoechoic, most commonly concentric arterial wall thickening. Key words: Giant cell arteritis, Polymyalgia rheumatica, Vasculitis, Ultrasonography.
\end{abstract}

Hevia J., et al. Utilidad de la ecotomografía Doppler en el diagnóstico de la arteritis de células gigantes Rev Chil Radiol 2018; 24(4): 134-141.

${ }^{*}$ Correspondencia: Joaquín Hevia M. / drjoaquinhevia@gmail.com

Trabajo enviado el 13 de septiembre de 2018. Aceptado para publicación el 27 de noviembre de 2018.

\section{Introducción}

La arteritis de células gigantes (ACG), también conocida como enfermedad de Horton, arteritis craneal o arteritis de la temporal, es la vasculitis sistémica más frecuente. Es una arteritis de adultos mayores, con una máxima incidencia en la séptima década de la vida, siendo virtualmente inexistente en menores de 50 años. La mayor parte de los síntomas y signos resultan del compromiso de ramas craneales de vasos supraaórticos, sin embargo, es una enfermedad sistémica que puede comprometer estructuras vasculares en cualquier parte del organismo ${ }^{(1,2,3)}$.En general es una enfermedad rápidamente progresiva que, sin el tratamiento adecuado, puede generar dolor severo, 
perdida visual permanente, lesiones cerebrovasculares e incluso la muerte. Todo lo anterior justifica la importancia de realizar un diagnóstico oportuno que permita el inicio precoz del tratamiento.

El diagnóstico de ACG debe ser considerado en pacientes mayores de 50 años con alguno de los síntomas o signos de la tabla 1. El antecedente o concomitancia de polimialgia reumática debe elevar la sospecha ante cualquiera de estos síntomas o $\operatorname{signos}^{(1)}$.

Tabla 1: Síntomas y signos para sospechar $\mathrm{ACG}^{*}$ en pacientes mayores de 50 años.

- Cefalea de inicio reciente.

- Inicio abrupto de alteraciones visuales, especialmente pérdida de visión monocular.

- Claudicación mandibular.

- Fiebre, anemia u otros síntomas constitucionales.

- Elevación de la velocidad de sedimentación de eritrocitos (VHS) y/o elevación de proteína-C reactiva (PCR).

${ }^{*} \mathrm{ACG}=$ Arteritis de células gigantes

Dado que el tratamiento con altas dosis de corticoides puede asociarse a riesgos significativos, el diagnóstico de ACG no se debe establecer sólo en base a criterios clínicos, debiendo basarse en resultados histopatológicos o imagenológicos que confirmen la enfermedad. La biopsia habitualmente se obtiene de la arteria temporal. Es posible también, en manos experimentadas, que la realización del estudio por ecotomografía-Doppler (ED) sustituya el estudio histopatológico en un contexto clínico apropiado (Figura 1).

\section{Biopsia de arteria temporal y sus limitaciones}

La biopsia de arteria temporal es un estudio imperfecto, existiendo falsos negativos explicados por la naturaleza segmentaria y heterogénea del compromiso de la pared arterial. Se han reportado tasas de falsos negativos de $29-44 \%$, probablemente relacionadas a las variadas definiciones clínicas de la enfermedad. Sin embargo, en ausencia de otro test diagnóstico definitivo, se considera el gold standard ${ }^{(1,4,5)}$. Una revisión bibliográfica que incluyó información de 2.986 pacientes, en que se consideró como gold standard el diagnóstico clínico o el resultado de biopsia unilateral o bilateral (si estaba disponible) de arteria temporal, demostró sensibilidad para biopsia unilateral de 86,9\% (IC 83,1-90,6)(6). Otros estudios han reportado sensibilidades tan bajas como $39 \%{ }^{(5)}$.

\section{Ecotomografía Doppler de arteria temporal}

El estudio ED de cabeza, cuello y/o extremidades superiores ha sido propuesto como alternativa diagnóstica a la biopsia de arteria temporal, con una especificidad reportada de $99,5 \%$ para la asociación de ED y evaluación clínica ${ }^{(7)}$. La ED tiene una resolución espacial de $0,1 \mathrm{~mm}$ con transductores de alta frecuencia ( $\geq 15 \mathrm{MHz}$ ), permitiendo la evaluación de arterias temporales y otras arterias extracraneales (Figura 2). En la ACG, las arterias temporales (u otras arterias craneales comprometidas) demuestran un área circunferencial hipoecogénica alrededor del lumen, probablemente determinada por infiltrados celulares y edema en la capa media, conocido como el signo del halo (Tabla 2) (Figuras 3a, 3b, 4a, 4d). Se han propuesto puntos de corte para detección de signo del halo (grosor parietal) entre 0,3 y $1,0 \mathrm{~mm}$ para arterias temporales y 1,0-2,0 $\mathrm{mm}$ para arterias axilares $^{(8)}$. Un estudio prospectivo reciente indica que el grosor del complejo medio-intimal normal mide hasta 0,2 $\mathrm{mm}$ en arteria temporal y $0,6 \mathrm{~mm}$ en arteria axilar y en pacientes con ACG estos alcanzan 0,5-0,8 $\mathrm{mm}$ y $1,5-2 \mathrm{~mm}$ respectivamente (Tabla 3$)^{(8,9)}$. Se ha descrito que la presencia de signo del halo bilateral y el signo de compresión (persistencia de visualización del halo durante la compresión con el transductor) son altamente específicos para ACG (Figuras 3d, $3 e)$. La presencia de estenosis u oclusión también pueden ser evidenciadas, pero tienen menor valor diagnóstico (Figuras 3c, 3d). En casos de estenosis se puede evidenciar elevación de velocidades al estudio Doppler-espectral, tomándose como punto de corte para estenosis hemodinámicamente significativas la duplicación de velocidades en sitio de estenosis.

Las ventajas del estudio ED incluyen ser un estudio no invasivo, sin irradiación y que además permite la evaluación de otras arterias (por ejemplo, axilares, occipitales o faciales), en caso de sospecha de compromiso de éstas. Los pacientes con compromiso de arteria facial tienen mayor frecuencia de claudicación mandibular y ceguera permanente ( 71 vs $27 \%$ y 24 vs $2 \%$ en pacientes con y sin compromiso respectivamente) ${ }^{(3)}$, por lo que puede ser útil explorar en forma dirigida esta arteria ante dicha sintomatología.

Los meta-análisis disponibles demuestran resultados variados de sensibilidad y especificidad de la técnica ED, con sensibilidades en rangos de $55-100 \%$ y especificidades de $78-100 \%$, comparados con biopsia de arteria temporal, considerando las limitaciones intrínsecas de este gold standard. Estudios recientes han demostrado que la ED tiene mayor sensibilidad que la biopsia(5,8). Se ha reportado que la presencia de signo del halo bilateral eleva la especificidad hasta un $100 \%{ }^{(10)}$. Algunos centros con mayores sensibilidades y especificidades reportadas han tendido a reemplazar la biopsia por estudio con ED como estudio inicial(8,10,11,12,13,14.15). La variabilidad 


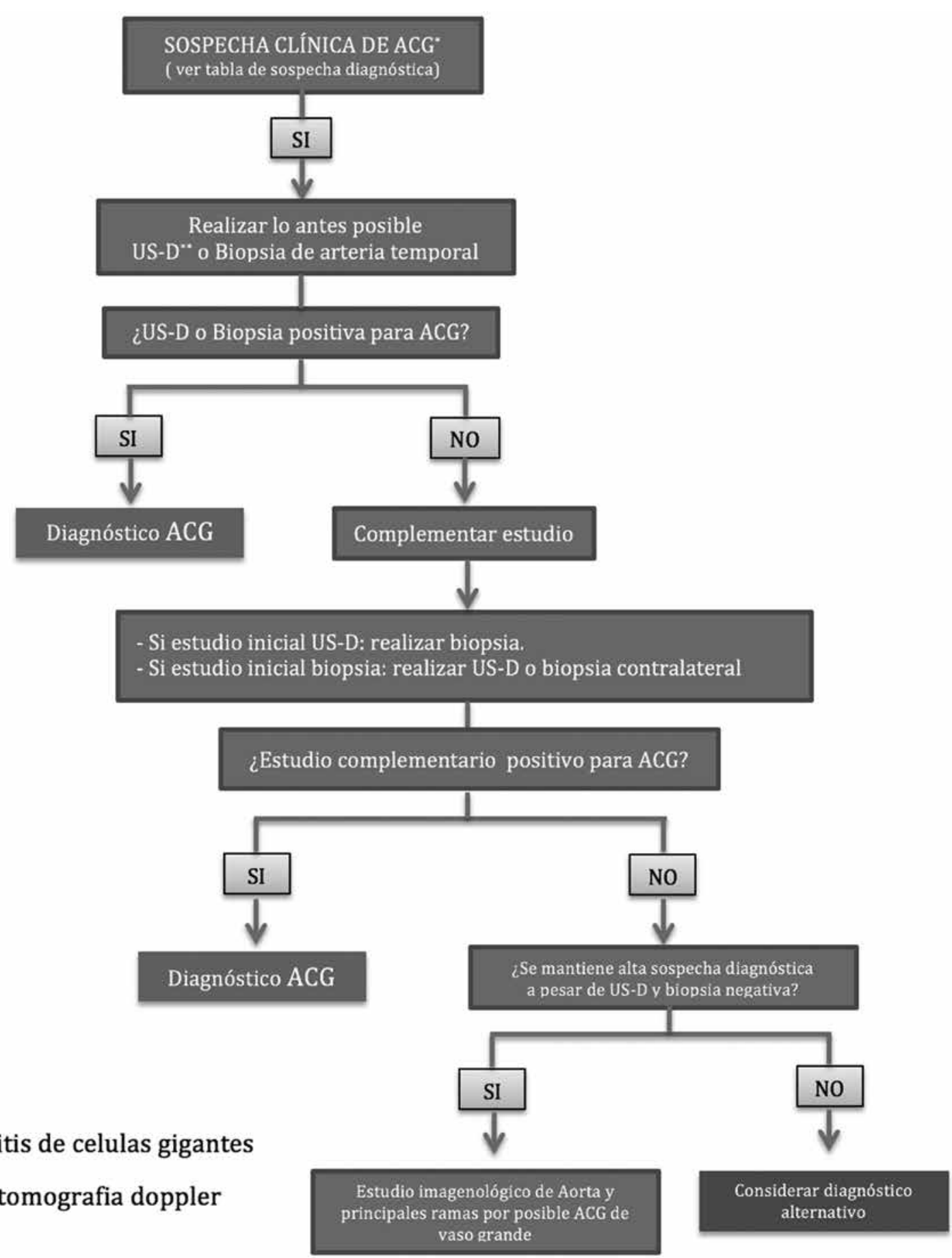

Figura 1: Algoritmo propuesto de diagnóstico en ACG. 

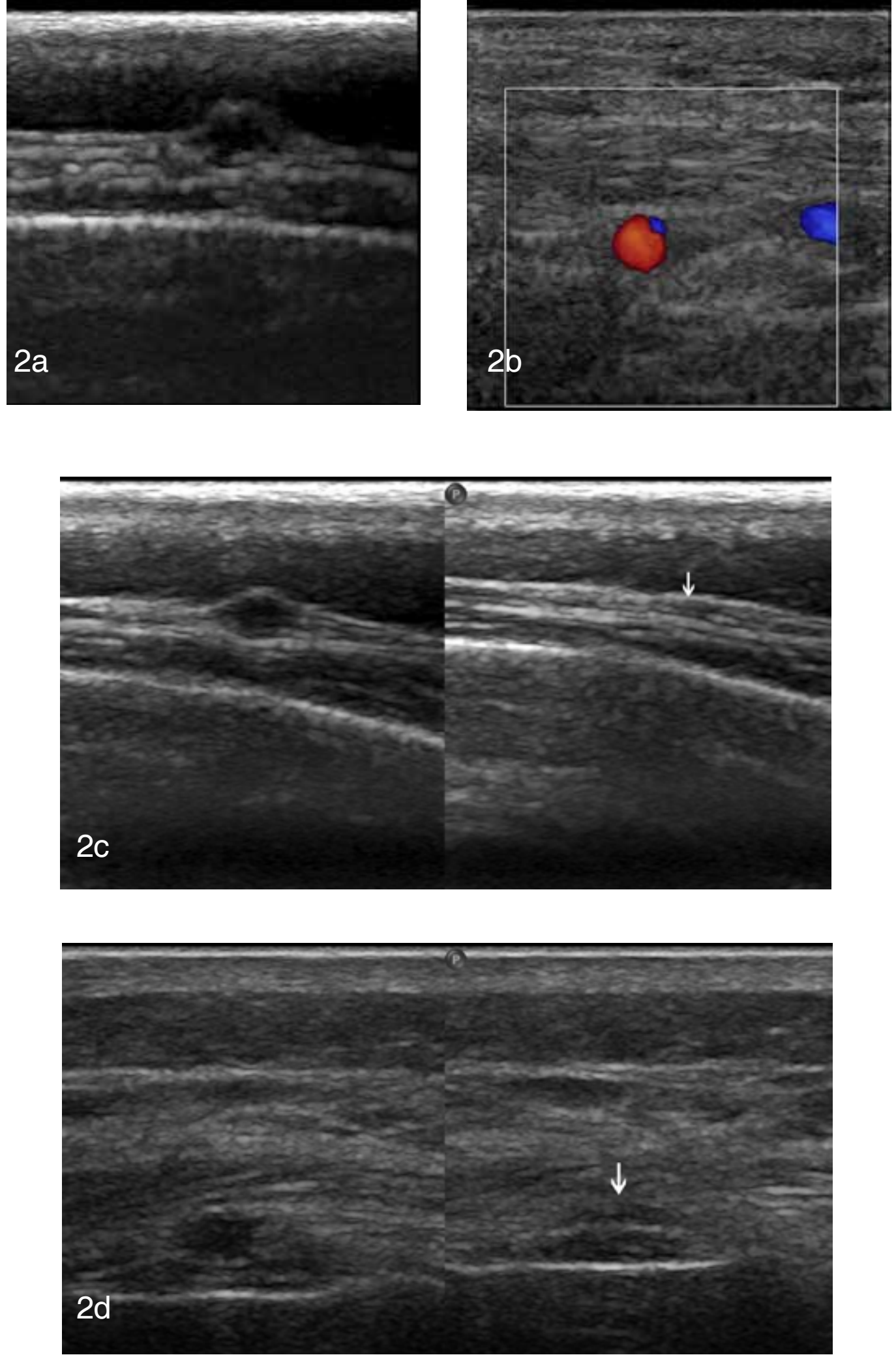

Figura 2: Arterias temporal y occipital normales. (a) Corte transversal en escala de grises de un segmento normal de arteria temporal. El lumen es anecogénico y las paredes arteriales son finas. (b) Corte transversal en modo Doppler-color en segmento normal de arteria temporal. El flujo llena la totalidad del lumen, observando paredes finas que rodean el área de flujo. Los parámetros deben estar calibrados para no subestimar ni sobreestimar el diámetro luminal. (c) Corte transversal en escala de grises de un segmento normal de arteria temporal sin y con compresión, donde se observa la compresibilidad total del lumen (flecha). En (d) se muestra la compresibilidad total (flecha) de una arteria occipital normal. 
Tabla 2: Signos de compromiso de arterias temporales en estudio por ecotomografía Doppler.

- Signo del halo (engrosamiento parietal circunferencial hipoecogénico)

- Signo de compresión (ausencia de compresibilidad)

- Estenosis

- Oclusión arterial
Tabla 3: Puntos de corte para evaluación de ACG en distintas arterias*.

Arteria temporal superficial común

$0,42 \mathrm{~mm}$

Rama frontal

$0,34 \mathrm{~mm}$

Rama parietal

$0,29 \mathrm{~mm}$

Arteria axilar

$1,0 \mathrm{~mm}$
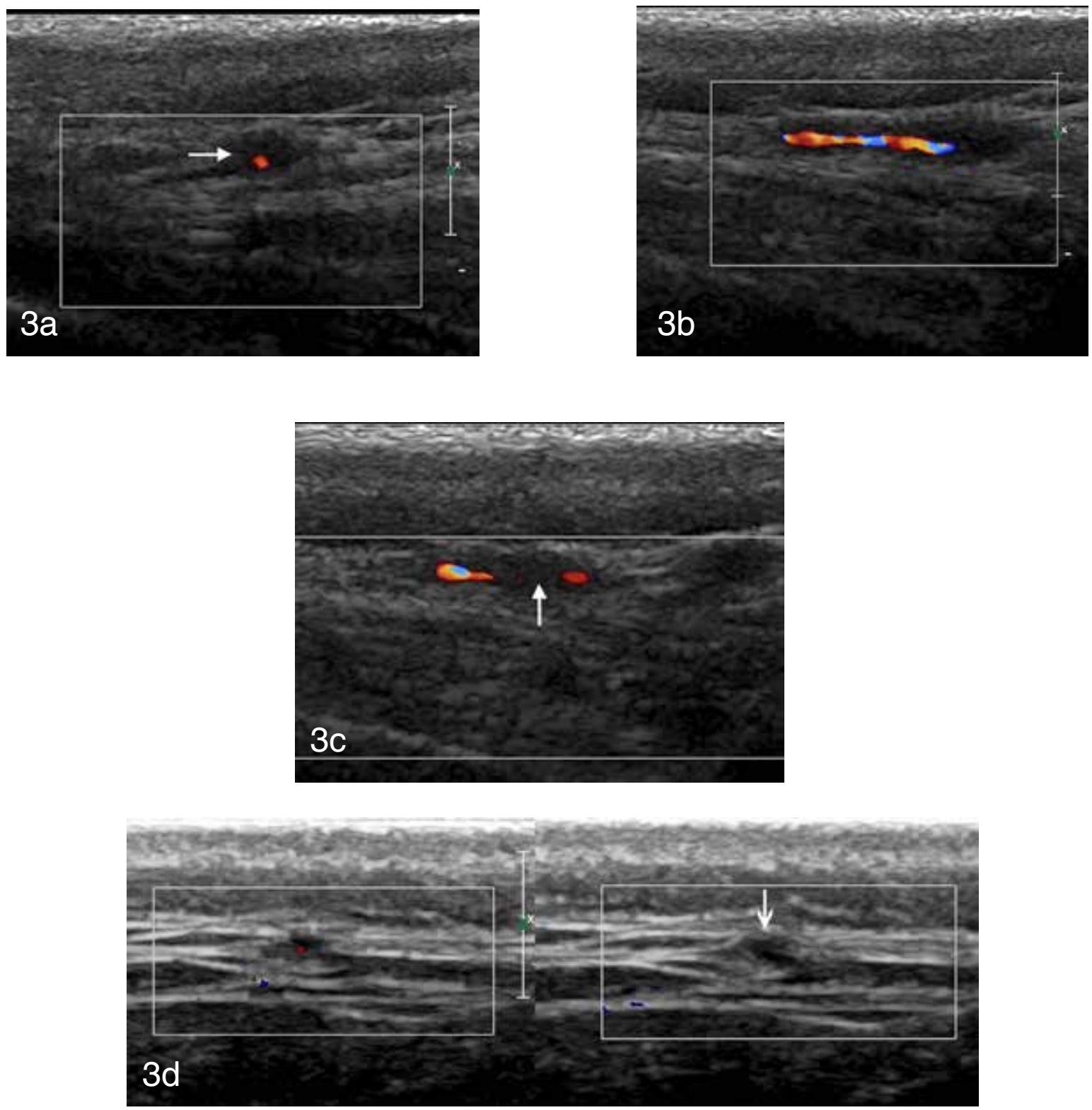

Figura 3: Paciente mujer de 69 años con arteritis de células gigantes. (a) Corte transversal de arteria temporal en modo Doppler color, que demuestra engrosamiento parietal circunferencial hipoecogénico compatible con signo del halo (flecha). (b) Corte longitudinal de arteria temporal en modo Doppler color, que demuestra un engrosamiento parietal irregular, visualizando en (c) un área de oclusión luminal focal (flecha). (d) Corte transversal de arteria temporal en modo Doppler color que demuestra estenosis luminal y signo del halo antes y después de la compresión, donde se demuestra la ausencia de compresibilidad (flecha) de la arteria temporal determinada por el engrosamiento hipoecogénico parietal. 
de las cifras y recomendaciones revelan la calidad de operador-dependiente de la ecotomografía. Se debe destacar además que el estudio debe ser realizado con un equipo y transductor de alta frecuencia $(\geq 15 \mathrm{MHz})$, que permitan una adecuada evaluación de las arterias temporales. Posiblemente los estudios más recientes demuestran mejores sensibilidades por la disponibilidad de mejor tecnología y existir mayor experiencia.

Existen distintos factores que pueden explicar los falsos negativos en la ED de arterias temporales. Un posible resultado negativo puede ocurrir en caso de paciente con ACG que tenga áreas de ausencia de compromiso (conocidas en inglés como "skip areas")(16). Otros potenciales falsos negativos ocurren en casos de compromiso vasculítico extracraneal, en que la arteritis se limita a grandes vasos (Figura 4), tendiendo a ser también negativas las biopsias de arteria temporal en estos casos (hasta $40 \%$ de los pacientes con ACG pueden no tener compromiso de arterias temporales) $(8,17,18,19)$.

Por estas razones se ha propuesto que el estudio de pacientes con sospecha de ACG debe incluir estudio con Ecotomografía Doppler de arterias temporales y
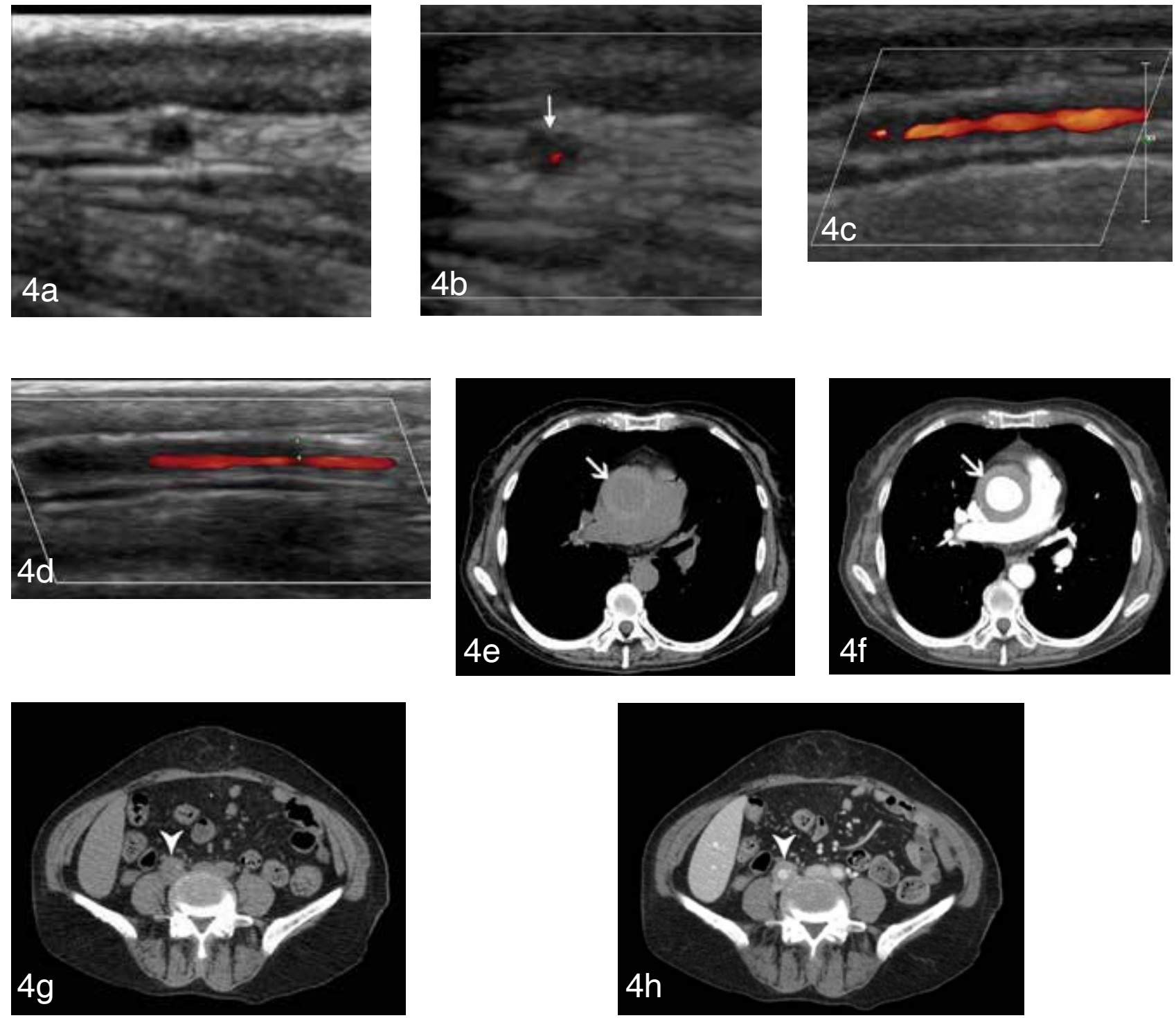

Figura 4: Paciente mujer de 75 años con arteritis de células gigantes (ACG). (a) Corte transversal de arteria temporal en escala de grises y en modo Doppler-color (b), que demuestran engrosamiento parietal circunferencial hipoecogénico compatible con signo del halo (flecha). (c) Corte longitudinal de arteria temporal en modo Doppler-color, que demuestra engrosamiento parietal irregular. En (d) se observa engrosamiento de borde liso de sus paredes. (e y f) Cortes axiales de tomografía computada (TC) sin y con contraste en ventana mediastínica, que demuestran marcado engrosamiento parietal circunferencial de aorta torácica ascendente (flechas), compatible con compromiso de grandes vasos por ACG en esta misma paciente. El compromiso se extendía también a lo largo de arteria ilíaca derecha, demostrados en cortes axiales de TC sin (g) y con (h) contraste (puntas de flecha). 
de arterias axilares. Ambas arterias temporales superficiales deben ser exploradas en forma continua, con transductores dedicados ( $\geq 15 \mathrm{MHz}$ ), incluyendo sus ramas frontal y parietal, tanto en plano transversal como longitudinal. Los pacientes con compromiso axilar son habitualmente más jóvenes, con promedio de 66 años, en comparación con 72 años en los casos de enfermedad craneal, siendo además más frecuente en mujeres ${ }^{(19)}$. Se puede incluir evaluación de arterias occipitales o faciales ante la sospecha clínica de compromiso de éstas ${ }^{(3,8)}$.La biopsia de arteria temporal tiene la desventaja de que evalúa sólo una pequeña zona anatómica en una enfermedad generalizada. Además de esto, el costo del procedimiento y el tiempo que transcurre en la entrega del resultado histológico es mayor. La ED permite evaluar el trayecto de múltiples arterias en un solo estudio, es de bajo costo y se realiza de forma rápida. Es por esto, que desde hace un tiempo se sugiere que la biopsia debe ser reservada para aquellos pacientes en que la ED arroje resultados inconsistentes con la evaluación clínica ${ }^{(20,21,22)}$.

Se debe tomar en cuenta que el tratamiento con corticoides reduce la sensibilidad de la ED. En un reporte de caso se describe la desaparición del signo del halo a los dos días de iniciada la terapia corticoesteroidal ${ }^{(23)}$ y se ha descrito que la sensibilidad disminuye a un $50 \%$ luego del cuarto día ${ }^{(24)}$.

\section{Conclusión}

El diagnóstico de ACG no debe basarse solamente en criterios clínicos, pudiendo ser certificado por biopsia o estudios de imágenes, siendo la biopsia de arteria temporal considerada aún como el gold standard, a pesar de sus limitaciones. En manos experimentadas, se puede implementar la ED como sustituto a la biopsia de arteria temporal, especialmente en casos en que se demuestren evidencias de compromiso bilateral con signo del halo.

Sería útil que los radiólogos demos a conocer la disponibilidad de la técnica en nuestros centros para aumentar nuestra experiencia y facilitar el diagnóstico no invasivo o guiar la biopsia en casos de sospecha de ACG. Quedará para estudios futuros la evaluación local de las sensibilidades y especificidades de esta técnica.

\section{Referencias}

1. Docken W. Diagnosis of giant cell arteritis. Trobe J, ed Waltham, MA UpToDate Inc http//www.uptodate. com (Accessed Sept 3, 2018).

2. González-Gay MA, García-Porrúa C. Systemic vasculitis in adults in Northwestern Spain, 1988-1997: Clinical and epidemiologic aspects. Medicine (Baltimore). 1999; 78(5): 292-308. doi: 10.1097/00005792199909000-00002.

3. Ješe R, Rotar Ž, Tomšič M, Hočevar A. The role of colour doppler ultrasonography of facial and occipital arteries in patients with giant cell arteritis: A prospective study. Eur J Radiol. 2017 (February); 95: 9-12. doi: 10.1016/j.ejrad.2017.07.007.

4. Duhaut $P$, Pinède $L$, Bornet $\mathrm{H}$, et al. Biopsy proven and biopsy negative temporal arteritis: $\mathrm{Di} V$ erences in clinical spectrum at the onset of the disease. Ann Rheum Dis. 1999; 58: 335-341.

5 Luqmani R, Lee E, Singh S, et al. Temporal Arteries in the Diagnosis and Treatment. 2016; 20(90). doi:10.3310/hta20900.

6. Niederkohr RD, Levin LA. Management of the patient with suspected temporal arteritis: A decision-analytic approach. Ophthalmology. 2005; 112(5): 744-756. doi: 10.1016/j.ophtha.2005.01.031.

7. Schmidt WA, Kraft HE, Vorpahl K, Völker L, GromnicaIhle EJ. Color Duplex Ultrasonography in the Diagnosis of Temporal Arteritis. N Engl J Med. 1997; 337(19): 1336-1342. doi: 10.1056/NEJM199711063371902.

8. Schmidt WA. Ultrasound in the diagnosis and management of giant cell arteritis. Rheumatology. 2018; 57(suppl_2): ii22-ii31. doi: 10.1093/rheumatology/ kex461.

9. Schäfer VS, Juche A, Ramiro S, Krause A, Schmidt WA. Ultrasound cut-off values for intima-media thickness of temporal, facial and axillary arteries in giant cell arteritis. Rheumatology, 2017: 56; 14791483. DOI: $10.1093 /$ rheumatology/kex143. Rheumatol (United Kingdom). 2017; 56: 1479-1483. doi: 10.1093/ rheumatology/kex289.

10. Arida A, Kyprianou M, Kanakis M, Sfikakis PP. The diagnostic value of ultrasonography-derived edema of the temporal artery wall in giant cell arteritis: A second meta-analysis. BMC Musculoskelet Disord. 2010; 11. doi:10.1186/1471-2474-11-44.

11. Duftner C, Dejaco C, Sepriano A, Falzon L, Schmidt WA, Ramiro S. Imaging in diagnosis, outcome prediction and monitoring of large vessel vasculitis: A systematic literature review and meta-analysis informing the EULAR recommendations. 2018. doi: 10.1136/rmdopen-2017-000612.

12. Ball EL, Walsh SR, Tang TY, Gohil R, Clarke JMF. Role of ultrasonography in the diagnosis of temporal arteritis. 2010: 1765-1771. doi: 10.1002/bjs.7252.

13. Karassa FB, Matsagas MI, Schmidt WA, Ioannidis JPA. Review Meta-Analysis: Test Performance of Ultrasonography for. Ann intern Med. 2005; 142: 359-369. doi: 10.7326/0003-4819-142-5-20050301000011.

14. Dejaco C, Duftner C, Buttgereit F, Matteson EL, Dasgupta B. Review The spectrum of giant cell arteritis and polymyalgia rheumatica: Revisiting the concept of the disease. Reumatology 2017; 506-515. doi: 10.1093/rheumatology/kew273.

15. Review C. Polymyalgia Rheumatica and Giant Cell Arteritis A Systematic Review. JAMA 2016; 315(22): 2442-2458. doi: 10.1001/jama.2016.5444.

16. Burke A, Virmani R. Temporal artery biopsy of giant cell arteritis. Pathol Case Rev. 2001; 6(6): 265-273. doi: 10.1097/00132583-200111000-00005.

17. Brack A, Martinez-Taboada V, Stanson A, Goronzy $\mathrm{JJ}$, Weyand CM. Disease pattern in cranial and largevessel giant cell arteritis. Arthritis Rheum. 1999; 42(2): 
311-317. doi:1 0.1002/1529-0131(199902)42:2<311::AIDANR14>3.0.CO;2-F.

18. Czihal M, Zanker S, Rademacher A, et al. Sonographic and clinical pattern of extracranial and cranial giant cell arteritis. Scand J Rheumatol. 2012; 41(3): 231-236. doi: 10.3109/03009742.2011.641581.

19. Schmidt WA, Seifert A, Gromnica-ihle E, Krause A, Natusch A. Ultrasound of proximal upper extremity arteries to increase the diagnostic yield in large-vessel giant cell arteritis. Rheumatology. 2008; 47(1): 96-101. doi: 10.1093/rheumatology/kem322.

20. Alberts MS, Mosen DM. Diagnosing temporal arteritis: Duplex vs. biopsy. Qjm. 2007; 100(12): 785-789. doi: 10.1093/qjmed/hcm103.

21. Diamantopoulos AP, Haugeberg G, Hetland H, Soldal DM, Bie R, Myklebust G. Diagnostic value of color Doppler ultrasonography of temporal arteries and large vessels in giant cell arteritis: A consecutive case series. Arthritis Care Res. 2014; 66(1): 113-119. doi:10.1002/acr.22178.

22. Karahaliou M, Vaiopoulos G, Papaspyrou S, Kanakis MA, Revenas K, Sfikakis PP. Colour duplex sonography of temporal arteries before decision for biopsy: A prospective study in 55 patients with suspected giant cell arteritis. Arthritis Res Ther. 2006; 8(4): 1-8. doi: 10.1186/ ar2003.

23. Clinical vignette. 2010: 2007. doi: 10.1093/rheumatology/ kes387.

24. Hauenstein C, Reinhard M, Geiger J, et al. Concise report Effects of early corticosteroid treatment on magnetic resonance imaging and ultrasonography findings in giant cell arteritis. 2012(July); 1999-2003. doi: 10.1093/ rheumatology/kes153. 